\title{
Influência da vinificação em talha na composição físico-química e fenólica de vinho tinto de região tropical
}

\author{
Influence of vinification in carving on the physicochemical and phenolic composition of red wine \\ from tropical region \\ Influencia de la vinificación tallada en la composición fisicoquímica y fenólica del vino tinto tropical
}

Recebido: 14/07/2021 | Revisado: 21/07/2021 | Aceito: 24/07/2021 | Publicado: 31/07/2021

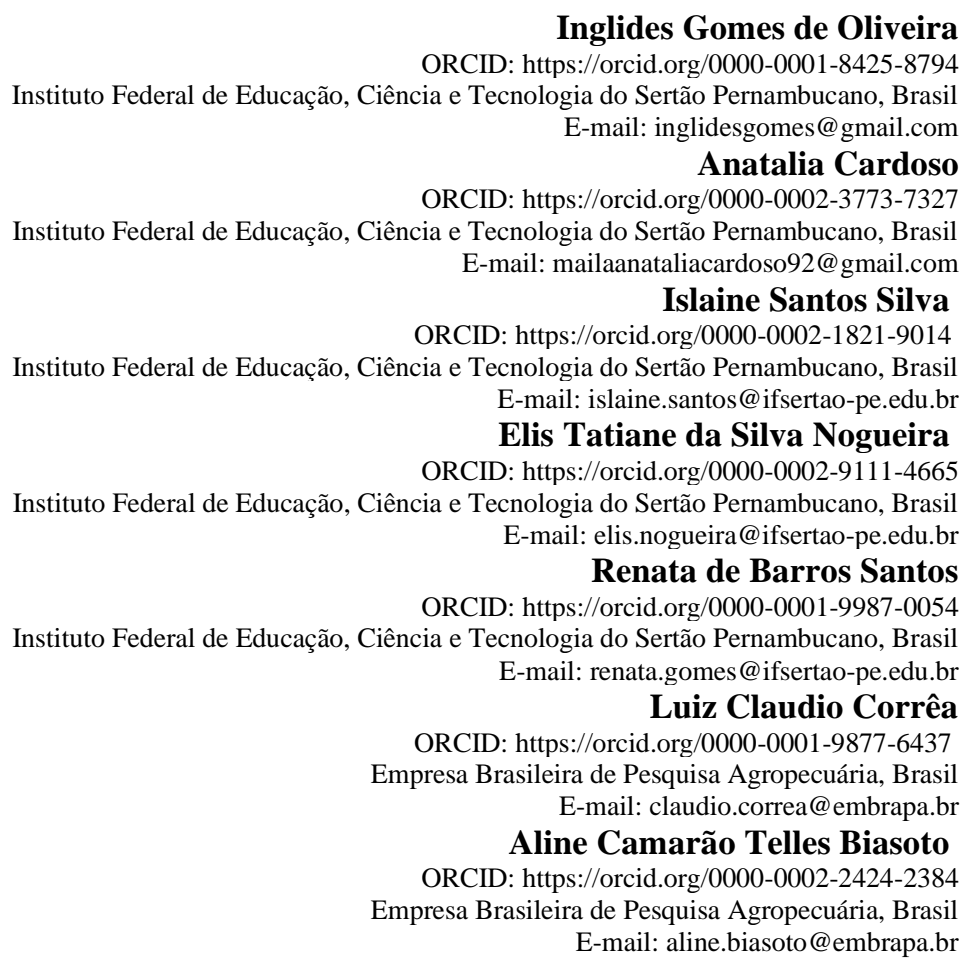

\section{Resumo}

A vitivinicultura tropical tem se expandido nos últimos anos, graças a cultivares adaptáveis ao clima, possibilitando à região do Submédio do Vale do São Francisco ser a segunda maior produtora de vinhos finos do Brasil, com destaque para cultivar Syrah, com boa adaptabilidade e elevados vigor e produtividade. Uma forma alternativa para a elaboração de vinhos utiliza ânforas, recipientes cerâmicos porosos revestidos com diferentes materiais, possibilitando a obtenção de vinhos diferenciados devido ao contato durante a fermentação. O presente trabalho tem como objetivo avaliar a influência do uso de garrafões de vidro e talhas de barro, na composição físico-química e fenólica de vinhos tintos Syrah elaborados no Submédio de Vale do São Francisco. Foram testados dois tratamentos, uma vinificação em garrafões de vidro e o outro vinificação em talhas de barro. Os parâmetros avaliados foram: pH, acidez total e volátil, açúcares redutores, teor alcoólico, índice de polifenóis totais (IPT), intensidade de cor, tonalidade, compostos fenólicos isolados e antocianinas monoméricas totais. Os resultados mostraram que não houve variação significativa entre os tratamentos estudados com relação aos parâmetros físico-químicos e colorimétricos, ficando os resultados dentro do estabelecido na legislação. As diferenças significativas encontradas entre os compostos fenólicos isolados e antocianinas monoméricas totais não influenciaram negativamente nos padrões da qualidade do vinho.

Palavras-chave: Vinificação artesanal; Vinhos tropical; Polifenóis; Submédio do Vale do São Francisco.

\begin{abstract}
Tropical viticulture has expanded in recent years, thanks to cultivars adaptable to the climate, enabling the Submiddle region of the São Francisco Valley to be the second largest producer of fine wines in Brazil, with emphasis on cultivating Syrah, with good adaptability and high vigor and productivity. An alternative way to prepare wines is to use amphorae, porous ceramic receptors coated with different materials, enabling the obtainment of differentiated wines due to contact during fermentation. The present work aims to evaluate the use of glass demijohns and clay pots
\end{abstract}


in the physical-chemical and phenolic composition of red Syrah wines produced in the Submédio region of Vale do São Francisco. Two treatments were tested, one vinification in glass demijohns and the other vinification in clay pots. The parameters were: $\mathrm{pH}$, total and volatile acidity, reducing sugars, alcohol content, total polyphenol index (TPI), color intensity, hue, previous phenolic compounds and total monomeric anthocyanins. The results inherent that there was no limited variation between the treatments studied with respect to physicochemical and colorimetric parameters, the results being within what is established in the legislation. The differences found between accumulated phenolic compounds and total monomeric anthocyanins do not negatively influence negatively in the wine quality standards.

Keywords: Craft winemaking; Tropical wines; Polyphenols; Middle of the São Francisco Valley.

\section{Resumen}

La viticultura tropical se ha expandido en los últimos años, gracias a cultivares adaptables al clima, permitiendo a la región Submedia del Valle de São Francisco ser el segundo productor de vinos finos en Brasil, con énfasis en el cultivo de Syrah, con buena adaptabilidad y alto vigor y productividad. Una forma alternativa de elaborar vinos son las ánforas, recipientes de cerámica porosa recubiertos con diferentes materiales, que permiten obtener vinos diferenciados por contacto durante la fermentación. El presente trabajo tiene como objetivo evaluar la influencia del uso de damajuanas de vidrio y vasijas de barro en la composición fisicoquímica y fenólica de los vinos tintos Syrah producidos en la región Submédio de Vale do São Francisco. Se probaron dos tratamientos, una vinificación en damajuanas de vidrio y la otra vinificación en vasijas de barro. Los parámetros evaluados fueron: $\mathrm{pH}$, acidez total y volátil, azúcares reductores, contenido de alcohol, índice de polifenoles totales (IPT), intensidad de color, tonalidad, compuestos fenólicos aislados y antocianinas monoméricas totales. Los resultados mostraron que no hubo variación significativa entre los tratamientos estudiados en relación a los parámetros físico-químicos y colorimétricos, estando los resultados dentro de los límites establecidos en la legislación. Las diferencias significativas encontradas entre los compuestos fenólicos aislados y las antocianinas monoméricas totales no influyeron negativamente en los estándares de calidad del vino.

Palabras clave: Elaboración de vinos artesanales; Vinos tropicales; Polifenoles; Medio del Valle de São Francisco.

\section{Introdução}

O Brasil vem se mostrando como produtor de boas safras de vinhos como crescimento no mercado vitivinicultor nacional e internacional nos últimos anos, apresentando em seu avanço vinhos da região tropical (Martin \& Nicoletti, 2016). Tradicionalmente, as regiões de clima temperado são favoráveis ao cultivo da videira, obtendo uva de qualidade para elaboração de vinho, porém, várias cultivares são adaptáveis também a regiões de clima tropical (Padilha et al., 2017).

O Submédio do Vale do São Francisco localizado no nordeste brasileiro é uma região de clima tropical. De acordo com Biasoton et al. (2014), é a segunda maior região produtora de vinhos finos do Brasil, com cerca de 500 hectares de videiras de uvas Vitis vinifera L, que produzem ao redor de 6 milhões de litros de vinho/ano, sendo 30\% elaborado com a cultivar Syrah. A região apresenta características edafoclimáticas que possibilitam o escalonamento da produção de uvas para a elaboração de vinhos durante todo o ano, além disso tais características também favorecem a produção de duas safras por ano, se tornando um diferencial dentre as outras regiões do Brasil que possuem uma safra por ano (Pereira et al., 2018).

A Syrah é considera uma das castas que mais se adaptou na região do Vale do São Francisco e, também é considerada a mais antiga variedade, podendo ser encontrada principalmente no Vale do Rhône (Fraga, 2010). É uma uva fácil de cultivar muito vigorosa e produtiva. Possui um curto período de maturação e revela-se bastante sensível à podridão no final da maturação. Origina vinhos de coloração intensa, aromáticos, finos e complexos, aptos ao envelhecimento e de grande qualidade (Jackson, 2000).

Historicamente os vinhos são elaborados em diversos recipientes, sendo utilizados, tanques de aço inoxidável, cubas de cimento, barris de madeira, ou em talha de barro ou cerâmica. Os recipientes devem ser neutros, e cada um fornecerá uma particularidade a bebida no final (Milan, 2019). A utilização de barricas de madeira durante a elaboração de vinhos tem como objetivo principal melhorar as características sensoriais da bebida (Jackson, 2014). Segundo Montalvo et al. (2021) os vinhos elaborados em tanques de aço inox são considerados mais estáveis e homogêneos com relação aos aspectos de aromas e sabor. Já os vinhos provenientes de tanques de concreto possuem potencial sensorial diferenciado devido a incorporação de oxigênio devido ao contato da bebida e o concreto (Miller; Oberholster \& Block, 2019). 
Os Vinhos de Talha, é conhecida tecnologia milenar, em que a fermentação alcoólica da bebida ocorre em vasos de barro. As talhas definem-se, em princípio, como um vaso-recipiente cerâmico, mais ou menos poroso de acordo com o tipo de argila de que é feito, e apresentando formas mais redondas ou mais alongadas, que são destinados ao armazenamento e transporte de produtos líquidos (Funari, 2007). Devido ao processo de elaboração das talhas, estas apresentam porosidade diferenciada, podendo tornar a qualidade e/ou tonalidade dos vinhos completamente diferentes (Caveira, 2018).

Os vinhos de talha são elaborados deixando em contado o mosto (líquido) e casca (sólido) durante todo o processo de fermentação (Santos, 2018). Durante esta etapa ocorre a transferência de diversos constituintes, dentre eles, os compostos fenólicos, que são responsáveis principalmente pela coloração e sabor no vinho (Garcìa-Marino et al., 2013). Esses compostos incluem os ácidos fenólicos, estilbenos, os flavonóis, flavanóis e as antocianinas. (Lins \& Sartori, 2014).

Os compostos fenólicos produzidos na uva são classificados em duas classes, são eles flavonóides e não flavonoides, a primeira classe corresponde principalmente as catequinas, epicatequinas, epigalocatequina, caempferol, quercetina, miricetina e antocianinas. A segunda classe engloba os ácidos fenólicos como os ácidos hidroxibenzóico e hidroxicinâmico, e os estilbenos (krikorian et al., 2012; Toaldo et al., 2016; Corredor et al., 2016; Toscano et al., 2017).

Os elementos constituintes do vinho são o resultado das substâncias presentes na uva, bem como da fermentação alcoólica. As características de um vinho estão, portanto, dependentes da casta utilizada, da qualidade da vindima, condições ambientais e técnicas de vinificação (Lins \& Sartori, 2014). Dessa forma, a composição fenólica dos vinhos influencia também, em suas variáveis físico-químicas, diretamente relacionadas com o padrão de qualidade normatizados para a bebida.

Os vinhos de talha são elaborados com práticas enológicas ancestrais, considerados diferente do atual processo de vinificação. A utilização de talhas de barro e o maior tempo de maceração durante a elaboração dos vinhos, contribuem para o desenvolvimento de características sensoriais diferenciadas. Não existem dados na literatura sobre a influência das talhas com relação a composição dos vinhos no nordeste brasileiro. Para resolver este problema, o presente trabalho tem como objetivo avaliar a influência do uso de garrafões de vidro e talhas de barro, na composição físico-química e fenólica de vinhos tintos Syrah elaborados no Submédio de Vale do São Francisco.

\section{Metodologia}

\subsection{Local do experimento}

Os vinhos foram elaborados no Instituto Federal de Educação, Ciência e Tecnologia do Sertão Pernambucano, Campus Petrolina Zona Rural, onde foram realizadas as análises clássicas e colorimétricas. Amostras dos vinhos foram enviadas ao Laboratório de Enologia da Embrapa Semiárido, Petrolina, PE, onde foram realizadas as análises de compostos fenólicos isolados e Antocianinas Monoméricas Totais.

\subsection{Delineamento experimental}

Os vinhos foram elaborados pelo método tradicional (Peynaud, 1997), utilizando-se uvas da c.v Syrah, doada pela vinícola Terra Nova do Grupo Miolo, localizada em Santana do Sobrado, município de Casa Nova, Bahia, Brasil.

Os tratamentos consistiram na elaboração de vinhos tintos, um microvinificado em garrafões de vidro e o outro microvinificado em talhas de barro, conforme descrito na Tabela 1. As vinificações foram realizadas em triplicata. 
Tabela 1 - Descrição dos tratamentos.

\begin{tabular}{ll}
\hline Tratamentos & Descrição \\
\hline Com garrafões (VG) & Vinificação em garrafões de vidro. \\
Com talhas (VT) & Vinificação em talhas de barro. \\
\hline
\end{tabular}

Fonte: Autores (2021).

\subsection{Determinações analíticas}

\subsubsection{Parâmetros físico-químicos}

As análises físico-químicas e colorimétricas foram realizadas no Laboratório de Enologia da Embrapa Semiárido, Petrolina, Pernambuco, Brasil. Seguindo procedimentos da Association of official analytical chemists - AOAC (2005), foram determinados nos vinhos o potencial hidrogeniônico $(\mathrm{pH})$, acidez total, acidez volátil, teor alcoólico, extrato seco, densidade, teor de dióxido de enxofre livre e total e os açúcares redutores residuais. O índice de Polifenóis Totais (IPT) foi determinado pelo procedimento descrito por Habertson e Spayd (2006). Todas as análises foram realizadas em triplicata para cada tratamento empregado.

\subsubsection{Análise instrumental da cor}

A intensidade de cor (IC) e tonalidade foram determinadas a partir da leitura das absorbâncias nos comprimentos de 420nm, 520nm e 620nm em espectrofotômetro ThermoFisher Scientific ${ }^{\circledR}$ (modelo Multiskan Go, Massachusetts, EUA), segundo método de Ough e Amerine (1988).

\subsubsection{Antocianinas Monoméricas Totais}

Seguindo metodologia da AOAC, (2005), o teor de antocianinas monoméricas totais foi estimado pelo método do $\mathrm{pH}$ diferencial, que tem a vantagem de considerar as transformações estruturais do cromóforo antocianina em função do pH. Por este método, a absorbância da amostra é medida a pH 1,0 (antocianinas como sais de oxônio coloridas), bem como a pH 4,5 (antocianinas como hemicetais incolores). As amostras foram previamente diluídas em tubos de $10 \mathrm{~mL}(1 \mathrm{~mL} \mathrm{de} \mathrm{vinho} \mathrm{+} 9 \mathrm{~mL}$ de metanol acidificado a 0,1\% com $\mathrm{HCl}$ ). Para cada tratamento, foram utilizados 6 tubos, sendo pipetado $1 \mathrm{~mL}$ da amostra diluída em cada tubo. Em seguida, para 3 dos tubos, foram pipetados $4 \mathrm{~mL}$ de tampão pH 1,0 e para os outros tubos, $4 \mathrm{~mL}$ de tampão pH 4,5. Após agitação da mistura em vórtex por 5 segundos, foram realizadas as leituras em espectrofotômetro, nos comprimentos de onda de 520nm e 700nm, zerados com água destilada. O teor das antocianinas monoméricas foi expresso em $\mathrm{mg} / \mathrm{L}$ de malvidina-3-O glucosídeo.

\subsubsection{Compostos fenólicos isolados por Cromatografia Líquida de Alta Performance (HPLC).}

Vinte e um compostos fenólicos foram quantificados nas amostras de vinho utilizando a metodologia previamente validada por Natividade et al., (2013). O equipamento utilizado foi um cromatógrafo líquido, modelo e2675, acoplado a um detector DAD, modelo 2475, ambos da Waters. Para a separação dos compostos, empregou-se uma coluna Gemini-NX $3 \mu$ C18(150 x 4,6 mm), e a pré-coluna Gemini-NXC18 (4,0 x 3,0 mm), ambas da marca Phenomenex® (USA), nas condições descritas pelo método. As amostras de vinho foram previamente filtradas em membrana de $0,45 \mu \mathrm{m}$ para vials, sendo injetados $10 \mu \mathrm{L}$ em triplicata. Foram detectados e quantificados, os seguintes compostos: a 280nm (DAD), ácido gálico, cis- resveratrol a 320nm (DAD), trans-resveratrol, piceatanol, viniferina, ácido caféico, ácido caf tarico, ácido ferrúlico, ácido cumarico e ácido clorogênico; a $360 \mathrm{~nm}$ (DAD), caempferol-3-O-glicosídeo, miricetina, quercetina-3-B-D-glucosideo, rutina e 
isorhamnetina-3-O-glicosídeo; a $520 \mathrm{~nm}$ (DAD), malvidina-3-O-glicosídeo, peonidina-3-O-glicosídeo, delfinidina-3-Oglicosídeo e pelargonidina-3-O-glicosídeo, petunidina-3-O-glicosídeo.

\subsection{Análise estatística}

A análise estatística foi realizada om auxílio do com o auxílio do programa XLstat ${ }^{\circ}$ versão 2015. Os resultados foram submetidos à ANOVA e as médias comparadas pelo teste de Tukey $(\mathrm{p} \leq 0,05)$.

\section{Resultados e Discussão}

\subsection{Parâmetros físico-químicos}

Os resultados das análises físico-químicas dos vinhos resultantes dos tratamentos garrafões e talhas estão indicados na Tabela 2. A análise dos resultados demostra que, com exceção dos açúcares redutores, da acidez volátil e do teor alcoólico, entre os vinhos dos tratamentos empregados, não houve diferença significativa para todos os parâmetros analisados.

Tabela 2 - Resultados das análises físico-químicas dos vinhos.

\begin{tabular}{lcc}
\hline \multicolumn{1}{c}{ Parâmetros } & \multicolumn{2}{c}{ Tratamentos } \\
& VG & VT \\
\cline { 2 - 3 } Teor alcoólico $\left({ }^{\circ} \mathrm{GL}\right)$ & $16,0 \pm 0,094^{\mathrm{a}}$ & $14,3 \pm 0,489^{\mathrm{b}}$ \\
Acidez total $\left(\mathrm{g} \mathrm{L}^{-1}\right)$ & $7,03 \pm 0,064^{\mathrm{a}}$ & $7,033 \pm 0,064^{\mathrm{a}}$ \\
Acidez Volátil $\left(\mathrm{g} \mathrm{L}^{-1}\right)$ & $0,63 \pm 0,044^{\mathrm{b}}$ & $0,73 \pm 0,039^{\mathrm{a}}$ \\
Potencial hidrogênio $(\mathrm{pH})$ & $4,06 \pm 0,082^{\mathrm{a}}$ & $4,07 \pm 0,036^{\mathrm{a}}$ \\
Açúcares Redutores $\left(\mathrm{g} \mathrm{L}^{-1}\right)$ & $2,79 \pm 0,366^{\mathrm{b}}$ & $3,88 \pm 0,489^{\mathrm{a}}$ \\
\hline
\end{tabular}

VG: vinificação em garrafão; VT: vinificação em talha. Médias seguidas de letras diferentes, na linha, diferem entre si pelo teste de Tukey $(\mathrm{p}<0,05)$.

Fonte: Autores (2021).

O teor alcoólico dos vinhos elaborados apresentou diferença significativa, o valor variou entre 14,3 e $16^{\circ} \mathrm{GL}$. Essa diferença pode ser justificada pela característica de porosidade da ânfora, pode ter ocorrido à evaporação do álcool. Entretanto pode se afirmar que de acordo com a legislação brasileira o vinho dosdois tratamentos classifica-se como vinho tinto nobre por apresentar teor alcoólico entre 14,1 a $16^{\circ} \mathrm{GL}$ (Brasil, 2018).

O parâmetro da acidez volátil apresentou maior no vinho do tratamento de ânfora, essa alteração pode ser dada pelo maior contato com a presença de oxigênio presente nos recipientes. Contudo os valores encontrados para os dois tratamentos obedeceram ao limite preconizado pela legislação brasileira, que estabelece o limite máximo de acidez volátil de 1,2 g.L.-1 em ácido acético, sendo que na pesquisa realizada foi possível evidenciar o valor de $0,73 \mathrm{~g} . \mathrm{L}^{-1}$, atendendo asconformidades permitida pela legislação (Brasil, 2018).

O teor de açúcares redutores apresentou diferenças significativa entre os tratamentos dos vinhos elaborados em talhas e garrafões, o valor de açúcar encontrado variou entre 2,79 e 3,88 g.L¹, sendo que o tratamento do vinho com ânfora obteve maior concentração. Estes valores podem ser justificados pela característica de porosidade da ânfora, pois pode ter ocorrido à evaporação do álcool, indicam que os vinhos elaborados se apresentam secos, porque o açúcar residual se encontra abaixo de $4 \mathrm{~g} / \mathrm{L}^{-1}$ (Brasil, 2018). 


\subsection{Análise instrumental da cor}

Os resultados das análises de cor dos vinhos resultantes dos tratamentos garrafões (VG) e talhas (VT) estão indicados na Tabela 3 .

Tabela 3 - Resultados das análises de potencial colorimétrico dos vinhos.

\begin{tabular}{lcc}
\hline Parâmetros & \multicolumn{2}{c}{ Tratamentos } \\
\cline { 2 - 3 } & VG & VT \\
\hline IPT (Índice de Polifenóis Totais) & $71,7 \pm 1,7^{\mathrm{a}}$ & $64,8 \pm 2,5^{\mathrm{b}}$ \\
Intensidade de Cor (420+520+620 nm) & $14,97 \pm 0,57^{\mathrm{a}}$ & $14,06 \pm 0,70^{\mathrm{b}}$ \\
Tonalidade (420/520nm) & $0,87 \pm 0,03^{\mathrm{a}}$ & $0,87 \pm 0,04^{\mathrm{a}}$ \\
\hline
\end{tabular}

VG: vinificação em garrafão; VT: vinificação em talha. Médias seguidas de letras diferentes, na linha, diferem entre si pelo teste de Tukey $(\mathrm{p}<0,05)$.

Fonte: Autores (2021).

Diante dos resultados encontrados para a variante IPT, houve diferença estatística entre os tratamentos, os valores de IPT encontraram-se maiores em vinhos elaborados em garrafões, encontrando na quantidade de 71,7, justificados pela menor incorporação de oxigênio através do recipiente de vinificação. Uma presença maior de oxigênio pode fazer com que ocorra a oxidação das antocianinas.

Segundo Sapatinha (2015) o índice de polifenóis totais é um dos principais responsáveis pela percepção de cor no vinho, em conjuntura com dados trazidos por Silva et al. (2019), onde vinhos acima de 60 podem ser destinados a elaboração de vinhos de guarda, é perceptível a influência de IPT na qualidade final dos vinhos.

No parâmetro intensidade de cor os vinhos apresentaram diferenças significativas, sendo a cor mais intensa no vinho elaborado no garrafão de vidro $(14,97)$. O que pode ser justificado pela menor presença de oxigênio. Os vinhos vinificados em ânforas ocasionaram assim uma maior degradação da intensidade de cor nos vinhos, porém mantendo o pigmento responsável pela coloração dos vinhos.

\subsection{Antocianinas Monoméricas Totais}

O vinho obtido na vinificação em garrafão (VG) apresentou teor de antocianinas monoméricas de $226,6 \mathrm{mg} \mathrm{L}^{-1}$, valor $32 \%$ maior do que o encontrado na vinificação em talha (VT) (Tabela 4).

Tabela 4 - Teor de antocianinas monoméricas totais nas amostras.

\begin{tabular}{cc}
\hline Amostras & Antocianinas mg L $\mathbf{~}^{-1}$ \\
\hline VG & $226,6 \pm 2,23^{\mathrm{a}}$ \\
VT & $171,6 \pm 2,28^{\mathrm{b}}$ \\
\hline
\end{tabular}

VG: vinificação em garrafão; VT: vinificação em talha. Médias seguidas de letras diferentes, na linha, diferem entre si pelo teste de Tukey $(\mathrm{p}<0,05)$.

Fonte: Autores (2021).

Os menores teores de antocianinas encontrados nos vinhos elaborados em VT provavelmente tenham sido em decorrência da composição do recipiente, sendo a interação do vinho com o material com o qual as talhas foram elaboradas uma possibilidade, especialmente considerando-se que as mesmas foram revestidas com cera de abelha. Além disso, a ocorrência de microporos nas paredes do recipiente propicia o acúmulo de oxigênio, o que causa o aumento das reações de oxidação dos compostos fenólicos.

Embora o teor de antocianinas tenha sido menor no vinho elaborado em talha, o valor foi muito próximo aos teores encontrados por Carvalho, et., al (2020), que, estudando vinhos Syrah elaborados no Submédio Vale do São Francisco, 
provenientes de diferentes portas enxertos e safras, encontraram o valor médio de 181,41 $\mathrm{mg} \mathrm{L}^{-1}$. Além disso, os resultados foram maiores dos que os reportados por Cysneiros \& Vasconcelos (2015), que encontraram 101,96 $\mathrm{mg} \mathrm{L}^{-1} \mathrm{em}$ vinhos Syrah produzidos no Submédio Vale do São Francisco.

\subsection{Compostos fenólicos isolados por HPLC-DAD-FD}

Os teores de compostos fenólicos foram detectados em maior quantidade no tratamento VG para todas as classes. Entre os ácidos fenólicos, a diferença foi de $22 \%$, com destaques para o Ácido Caftárico, com 48,84 mg L $\mathrm{m}^{-1}$ em VG e 35,77 mg L ${ }^{-1}$ em VT, e Ácido Gálico, com 24,15 mg L-1 e 23,21 mg L-1 para VG e VT respectivamente (Tabela 5).

As concentrações de Ácido Caftárico foram superiores ao teor médio encontrado por Dias (2010) em três vinhos comerciais Syrah Submédio Vale do São Francisco $\left(29,06 \mathrm{mg} \mathrm{L}^{-1}\right)$. Já os teores de Ácido Gálico nos dois tratamentos foram maiores do que os encontrados por Carvalho, et al., (2020), cujos valores variaram entre 10,50 e 22,30 mg $\mathrm{L}^{-1}$. Segundo Angelo e Jorge (2007), os ácidos fenólicos estão presentes em especiarias, normalmente características pela presença de aromas destacados. Isso evidencia a importância dessa classe de compostos na composição aromática dos vinhos.

Tabela 5 - Quantificação de compostos fenólicos por HPLC-DAD-FD.

\begin{tabular}{|c|c|c|}
\hline \multirow{2}{*}{ Compostos fenólicos $\left(\mathrm{mg} \mathrm{L}^{-1}\right)$} & \multicolumn{2}{|c|}{ Amostras } \\
\hline & VG & VT \\
\hline \multicolumn{3}{|l|}{ Ácidos fenólicos } \\
\hline Ácido Gálico & $24,16^{\mathrm{a}}$ & $23,21^{\mathrm{b}}$ \\
\hline Ácido Cafeíco & $1,59^{\mathrm{a}}$ & $1,22^{b}$ \\
\hline Ácido Caftárico & $48,84^{\mathrm{a}}$ & $35,77^{b}$ \\
\hline Ácido Clorogênico & $0,45^{\mathrm{a}}$ & $0,38^{\mathrm{a}}$ \\
\hline Ácido Cumárico & $1,19^{\mathrm{b}}$ & $1,61^{\mathrm{a}}$ \\
\hline Ácido Ferrúlico & $0,48^{\mathrm{a}}$ & $0,44^{\mathrm{b}}$ \\
\hline \multicolumn{3}{|l|}{ Estilbenos } \\
\hline Piceatanol & $0,36^{\mathrm{a}}$ & $0,29^{b}$ \\
\hline Trans-Resveratrol & $0,35^{\mathrm{a}}$ & $0,33^{\mathrm{a}}$ \\
\hline Cis-Resveratrol & $0,41^{\mathrm{a}}$ & $0,46^{\mathrm{a}}$ \\
\hline Viniferina & $0,42^{\mathrm{a}}$ & $0,39^{\mathrm{b}}$ \\
\hline \multicolumn{3}{|l|}{ Flavonóis } \\
\hline Kaempferol-3-O-glucosideo & $1,78^{a}$ & $1,63^{\mathrm{b}}$ \\
\hline Quercetina-3-B-D-glucosideo & $31,29^{\mathrm{a}}$ & $27,16^{\mathrm{b}}$ \\
\hline Isorhamnetina-3-O-glucosideo & $16,40^{\text {a }}$ & $14,51^{\mathrm{b}}$ \\
\hline Miricetina & $1,03^{\mathrm{a}}$ & $0,95^{\mathrm{b}}$ \\
\hline Rutina & $1,29^{\mathrm{a}}$ & $1,00^{\mathrm{b}}$ \\
\hline \multicolumn{3}{|l|}{ Antocianinas } \\
\hline Pelargonidina-3-O-glucosideo & $6,10^{\mathrm{a}}$ & $3,10^{\mathrm{b}}$ \\
\hline Cianidina-3-O-glucosideo & $0,39^{\mathrm{a}}$ & $0,29^{\mathrm{b}}$ \\
\hline Delfinidina-3-O-glucosideo & $3,50^{\mathrm{a}}$ & $1,78^{\mathrm{b}}$ \\
\hline Malvidina-3-O-glucosideo & 44,03 a & $31,12^{b}$ \\
\hline Peonidina-3-O-glucosideo & $4,61^{\mathrm{a}}$ & $2,38^{\mathrm{b}}$ \\
\hline Petunidina-3-O-glucosideo & $0,88^{\mathrm{a}}$ & $0,85^{\mathrm{a}}$ \\
\hline
\end{tabular}

VG: vinificação em garrafão; VT: vinificação em talha. Médias seguidas de letras diferentes, na linha, diferem entre si pelo teste de Tukey $(\mathrm{p}<0,05)$.

Fonte: Autores (2021). 
Dentre os estilbenos, o Cis-Resveratrol foi o único que apresentou teor mais elevado em VT, com 0,46 $\mathrm{mg} \mathrm{L}^{-1}$, enquanto que em VG foram encontrados $0,41 \mathrm{mg} \mathrm{L}^{-1}$.

Com relação ao Trans-Resveratrol, os valores encontrados no presente trabalho, 0,33 $\mathrm{mg} \mathrm{L}^{-1}$ e $0,35 \mathrm{mg} \mathrm{L}^{-1}$ para VT e VG respectivamente, foram similares aos citados por Carvalho, et al., (2020), com teores variando entre 0,20 e $0,50 \mathrm{mg} \mathrm{L}^{-1}$.

Os estilbenos presentes em vinhos são comumente mencionados na literatura como compostos benéficos á saúde humana. Como destaque, pode-se citar o resveratrol, que atua prevenindo problemas cardíacos, sobre tudo, por sua ação antioxidante, além de atuar na resposta contra o ataque às videiras por fungos do tipo Botrytis cinerea, agindo como fitoalexina (Dias, 2010).

Com relação aos flavonóis, a diferença foi de $14 \%$ a mais para VG e os compostos majoritários foram a Quercetina-3-

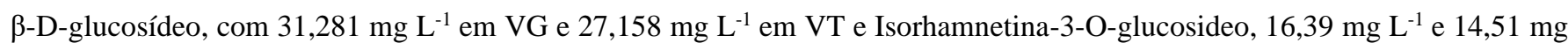
$\mathrm{L}^{-1}$, respectivamente. Esses teores foram maiores do que os valores médios encontrados por Carvalho, et al., (2020). Em seu estudo com vinhos Syrah provenientes de diferentes portas enxertos e safras, os autores reportaram teores médios de $20 \mathrm{mg} \mathrm{L}^{-1}$

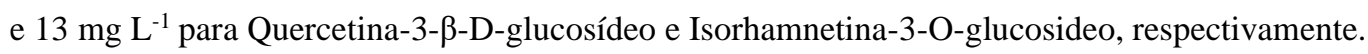

Os flavonóis são outro subgrupo dentro dos flavonoides. Nos vinhos tintos os principais flavonóis são a quercetina, miricetina e campferol. Estes componentes são encontrados nas cascas das uvas e extraídos durante a etapa de maceração dos vinhos tintos (Araújo, 2015). Segundo Rice-Evans, et al. (1996), a quercetina é o flavonóide que apresenta a maior atividade antioxidante no vinho.

A classe que apresentou maior diferença entre os tratamentos foi a das antocianinas. A soma apresentou o teor $50 \%$ maior em VG. O composto majoritário, Malvidina-3-O-glucosideo, correspondeu cerca de $76 \%$ do total, sendo encontrados, 44,026 mg L-1 em VG e 31,119 $\mathrm{mg} \mathrm{L}^{-1}$ VT, sendo este último, semelhante ao teor encontrado por Dias, (2010) em estudo com vinho Syrah comercial do Submédio Vale do São Francisco (32,20 $\left.\mathrm{mg} \mathrm{L}^{-1}\right)$.

O grupo das antocianinas é dividido em seis classes de compostos responsáveis pelas diferentes pigmentações: cianidina (vermelho), peonidina (vermelho escuro), delfinidina (azul), malvidina (púrpura) e petunidina (vermelho escuro) (Jackson, 1994). As antocianinas são compostos hidrossolúveis e termosensíveis, nas uvas estão concentradas principalmente nos vacúolos de células da casca. São um importante grupo de pigmentos responsáveis pelas tonalidades de vermelho e roxo características de uvas e vinhos. Os pigmentos antociânicos majoritários em uvas são a malvidina-3-glicosídio, petunidina-3glicosídio, cianidina-3-glicosídio, delfinidina-3-glicosídio, peonidina-3-glicosídio (Falcao, et al., 2007).

Os menores teores de compostos fenólicos encontrados nos vinhos elaborados em VT podem ser justificados, da mesma forma que as antocianinas, pelas interações com o material do recipiente, especialmente considerando-se que o mesmo foi impermeabilizado com cera de abelha, a qual é composta por uma mistura de ésteres (67\%), hidrocarbonetos (14\%), ácidos graxos (12\%), e álcoois (1\%) (Tulloch, 1980), Além disso, ocorrência de microporos nas paredes do recipiente propicia o acúmulo de oxigênio, o que causa o aumento das reações de oxidação dos compostos fenólicos. Segundo Salmon (2006), cerca de $30 \%$ do oxigênio é consumido na fermentação alcoólica do vinho, e destinado a vias oxidativas, sendo os compostos fenólicos, os primeiros a ser oxidados na presença de oxigênio, cuja capacidade de reação dependente da sua concentração (Karbowiak, et al., 2010).

\section{Conclusão}

As pequenas diferenças observadas na composição físico-química dos vinhos, devido, provavelmente à composição das talhas, não influenciaram negativamente nos padrões da qualidade do vinho, os quais se enquadraram dentro da legislação para vinhos finos nobres brasileiros, mantendo assim a qualidade após a conclusão da vinificação. 
Embora os teores de fenólicos tenham sido menores em talha, em ambos os casos os resultados se encontraram próximos aos citados na literatura. Considerando-se o menor custo para aquisição de talhas e o apelo artesanal envolvido no seu uso, o mesmo pode ser uma opção para pequenos produtores rurais que queiram investir na produção de vinhos sem a necessidade de grandes investimentos. Nesse sentindo, aditivamente, recomenda-se realizar análises complementares, bem como uma análise sensorial dos vinhos para avaliar de uma forma mais precisa a influência do recipiente analisado vínico na qualidade da bebida.

\section{Agradecimentos}

Ao Instituto Federal de Educação, Ciência e Tecnologia do Sertão Pernambucano (Campus Petrolina Zona Rural) pela concessão do espaço da Escola do Vinho para realização do experimento e análises, ao laboratório de enologia da Empresa Brasileira de pesquisa agropecuária (Embrapa Semiárido - Petrolina, Pernambuco, Brasil) pela realização das análises e à Vinícola Terranova - Miolo Wine Group pelo fornecimento das uvas.

\section{Referências}

Araújo, J. M. A. (2015). Química de alimentos: teoria e prática. (6a ed.), Ed. UFV.

Angelo, P. M., \& Jorge, N. (2007). Compostos fenólicos em alimentos-uma breve revisão. Revista do Instituto Adolfo Lutz (Impresso), 66(1), 01-09.

Association of official analytical chemists (2005). AOAC International The Scientific Association.

Baiano, A., Varva, G., De Gianni, A., Viggiani, I., Terracone, C., \& Del Nobile, M. A. (2014). Influence of type of amphora on physico-chemical properties and antioxidant capacity of 'Falanghina'white wines. Food chemistry, 146, 226-233.

Biasoto, A., Pereira, G., de Oliveira, J. B., de Menezes, T. R., \& Leao, P. D. S. (2014). Efeitos da desfolha e desponte de ramos sobre a composição físico química de Syrah elaborados em dois ciclos de produção no Vale do São Francisco. In Embrapa Semiárido-Artigo em anais de congresso. In: Congresso Brasileiro De Fruticultura, 23, Fruticultura: oportunidades e desafios para o Brasil. Cuiabá: SBF.

Brasil, Ministério da Agricultura e do Abastecimento. (2018). Complementação dos padrões de identidade e qualidade do vinho e dos derivados da uva e do vinho.

Caveira, J. P. G. (2018). Estudo para a Caracterização de Vinho de Talha na Região de Vila de Frades. Dissertação Mestrado em Engenharia Alimentar Instituto Politécnico de Beja, Escola Superior Agrária. Beja.

Cysneiros, G. F; Vasconcelos, M. A. S. (2015). Capacidade Antioxidante de Vinhos TropicaisBrasileiros.https://www.ufpe.br/docum. ents/616030/820209/Capacidade_antixidante.pdf

Coelho, A. P. (2017). Os Puro Talha de uma adega com mais de 100 ânforas. http://fugas.publico.pt/Vinhos/377477_os-purotalha-de-uma-adega-com-mais-de100-anforas.

Corredor, Z., Rodríguez-Ribera, L., Coll, E., Montañés, R., Diaz, J. M., Ballarin, J., \& Pastor, S. (2016). Unfermented grape juice reduce genomic damage on patients undergoing hemodialysis. Food and Chemical Toxicology, 92, 1-7.

De Carvalho, E. S. S., Biasoto, A. C. T., Nassur, R. D. C. M. R., Barros, A. P. A., Leão, P. C. S., da Silva Lima, R., \& de Oliveira Mamede, M. E. (2020). Physicochemical characteristics, phenolic profile, and antioxidant capacity, of Syrah tropical wines: Effects of vineyard management practices. Journal of Food Bioactives, 9 .

Dias, F. D. S. (2010). Determinação de compostos fenólicos em vinhos e caracterização de vinhos eleborados na região do vale do São Francisco Pernambuco. Tese (Doutorado em Ciências, área de concentração Química Analítica) - Universidade Federal da Bahia, Instituto De Química Programa De Pós-Graduação Em Química.

Falcão, A. P., Chaves, E. S., Kuskoski, E. M., Fett, R., Falcão, L. D., \& Bordignon-Luiz, M. T. (2007). Índice de polifenóis, antocianinas totais e atividade antioxidante de um sistema modelo de geléia de uvas. Food Science and Technology, 27(3), 637-642.

Fraga, K. de F. (2010). Aminas bioativas durante a maturação de uvas Syrah produzidas em diferentes regiões e sistemas de condução. Dissertação (Mestrado em Ciência de Alimentos) - Faculdade de Farmácia, Universidade Federal de Minas Gerias, Belo Horizonte.

Funari, P. P. A. (2007). Ânforas, registros do passado. História Viva, Grandes Temas, Vinho, 17, 18-21. https://www.academia.edu/10113786/\%C3\%82nforas_registros_do_passado_-_vinho

García-Marino, M., Escudero-Gilete, M. L., Heredia, F. J., Escribano-Bailón, M. T., \& Rivas-Gonzalo, J. C. (2013). Color-copigmentation study by tristimulus colorimetry (CIELAB) in red wines obtained from Tempranillo and Graciano varieties. Food Research International, 51(1), $123-131$.

Garrido, J., \& Borges, F (2013). Wine and grape polyphenols - A chemical perspective. Food Research International, 54, 1844-1858 
Harbertson, J, \& Spayd, S. (2006). Measuring phenolics in the winery. American Journal Enological and Viticultural, 57 (3), 280-288.

Jackson, R. (1994). Chemical Constituents of Grapes. In: WINE science: principles and applications. London: Academic Press, 178-219.

Jackson, R.S. (2000). Wine Science: principles, practice, perception. (2a ed.), Academic Press, 645p.

Jackson, R. S. (2014). Vineyard Practice. In: Wine Science. Elsevier, 143-306.

Karbowiak, T., Gougeon, R. D., Alinc, J. B., Brachais, L., Debeaufort, F., Voilley, A., \& Chassagne, D. (2009). Wine oxidation and the role of cork. Critical reviews in food science and nutrition, 50(1), 20-52.

Krikorian, R., Boespflug, E. L., Fleck, D. E., Stein, A. L., Wightman, J. D., Shidler, M. D., \& Sadat-Hossieny, S. (2012). Concord grape juice supplementation and neurocognitive function in human aging. Journal of agricultural and food chemistry, 60(23), 5736-5742.

Lins, A. R., \& Sartori, G. V. (2014). Qualidade fenólica e atividade antioxidante de vinhos tintos produzidos no estado do Paraná. Revista Brasileira de Produtos Agroindustriais, 16(1), 69-76.

Martins, P. P., Nicoletti, M. A. (2016). Polifenóis no vinho: resveratrol e seus benefícios. Infarma ciências farmacêuticas, 28(4), 216-225.

Milan, E. (2019). Adega. vinificação em ovos de concreto. https://revistaadega.uol.com.br/artigo/vinificacao-em-ovos-de-concreto_10353.html.

Miller, K. V., Oberholster, A., \& Block, D. E. (2019). Predicting fermentation dynamics of concrete egg fermenters. Australian journal of grape and wine research, 25(3), 338-344.

Montalvo, F. F., García-Alcaraz, J. L., Cámara, E. M, Jiménez-Macías, E., \& Blanco-Fernández, J. (2021). Impacto ambiental da fermentação do vinho em tanques de aço e concreto. Journal of Cleaner Production, 278, 123602.

Natividade, M. M. P., Correa, L.C., Souza, S. V. C., Pereira, G. E., Lima, L. C. O. (2013). Simultaneous analysis of 25 phenolic compounds in grape juice for HPLC: Method validation and characterization of Sao Francisco Valley samples. Microchemical Journal, 110.

Ough, C S, \& Amerine, M. A (1988). Métodos de análise de mostos e vinhos J. Wiley.

Padilha, C. V. D. S, Camarão Telles Biasoto, A., Corrêa, L. C, dos Santos Lima, M., \& Pereira, G. E (2017). Perfil de compostos fenólicos e atividade antioxidante de vinhos tintos tropicais comerciais (Vitis vinifera L.) do Vale do São Francisco, Brasil. Journal of Food Biochemistry, 41 (3), e12346.

Pereira, G. E., Guerra, C. C., de Amorim, F. M., Nascimento, A. D. S., de Souza, J. F., Lima, L. D. A., \& Tonietto, J. (2018). Vinhos tropicais do semiárido do Brasil: desvendando o potencial vitivinícola desta nova fronteira geográfica do vinho. Embrapa Uva e Vinho-Artigo em periódico indexado.

Peynaud, E. Connaissance et travail du vin. Editora Dunod, Paris, 1997.

Riberau-Gayon, P., Glories, Y., Maujean, A., \& Dubourdieu, D. (2006). Handbook of Enology. Volume 2: The Chemistry of Wine and Stabilization and Treatments. John Wiley \& Sons.

Rice-Evans, C. A., Miller, N. J., \& Paganga, G. (1996). Structure-antioxidant activity relationships of flavonoids and phenolic acids. Free radical biology and medicine, 20(7), 933-956.

Santos, A. R. J. (2018). Vinhos de talha. Caracterização química. Dissertação para a obtenção do Grau de Mestre em Viticultura e Enologia - Intituto Superior de Agronomia, Universidade de Lisboa.

Salmon, J. M. (2006). Interactions between yeast, oxygen and polyphenols during alcoholic fermentations: Practical implications. LWT-Food Science and Technology, 39(9), 959-965.

Sapatinha, M. M. G. (2015). Total polyphenol content of wine in contact with wood chips and subsequent colorimetric characteristics (Doctoral dissertation).

Silva, I., Barros, A., Nunes, G. D. S., Torres, L., do Nascimento, D. C., Damasceno, J., \& Biasoto, A. (2019). Influência da técnica de termovinificação no conteúdo de compostos fenólicos e na cor dos vinhos tintos tropicais Syrah. In Embrapa Semiárido-Artigo em anais de congresso (ALICE). In: Congreso Latinoamericano De Viticultura Y Enologia, 16, 2019, Ica, Peru. Desierto: pasión vitivinícola en Latinoamérica. Ica: Asociacion Peruana de Enologos: Organización Internacional de la Viña y del Vino, 2019.

Toaldo, I. M., Cruz, F. A., da Silva, E. L., \& Bordignon-Luiz, M. T. (2016). Acute consumption of organic and conventional tropical grape juices (Vitis labrusca L.) increases antioxidants in plasma and erythrocytes, but not glucose and uric acid levels, in healthy individuals. Nutrition Research, 36(8), 808-817.

Toscano, L. T., Silva, A S., Toscano, L. T., Tavares, R. L., Biasoto, A. C. T., de Camargo, A. C., \& Shahidi, F. (2017). Os fenólicos do suco de uva roxa aumentam o status antioxidante sérico e melhoram o perfil lipídico e a pressão arterial em adultos saudáveis sob intenso treinamento físico. Journal of Functional Food, 33 , 419-424.

Tulloch, A. P. (1980). Beeswax-composition and analysis. Bee World, 61 (2), 47-62. 